\title{
Intact proinsulin level is associated with insulin resistance but not insulin secretory capacity in subjects with abnormal glucose tolerance
}

You-Cheol Hwang, In-Jin Cho, In-Kyung Jeong, Kyu Jeung Ahn, Ho-Yeon Chung

Department of Endocrinology and Metabolism, Kyung Hee University School of Medicine, Seoul, Republic of Korea

\section{Introduction}

Proinsulin is a propeptide of insulin and C-peptide during physiological insulin production and is increased in patients with type 2 diabetes. Increased proinsulin in peripheral blood indicates impairment of cleavage capacity to process insulin within the beta cell. Recently, chemiluminescence assays have been developed that are able to specifically measure uncleaved intact proinsulin in peripheral blood. In this cross-sectional study, we investigated the relationship between intact proinsulin and insulin resistance and secretion in Korean adults.

\section{Materials and Methods}

We performed standard $75 \mathrm{~g}$ oral glucose tolerance test (OGTT) after an overnight fast in 388 subjects without history of diabetes. Glucose $(0,30$, $60,90,120 \mathrm{~min})$, insulin $(0,30,60,90,120 \mathrm{~min})$, C-peptide (0, $30 \mathrm{~min})$, and fasting intact proinsulin were measured and insulin sensitivity and secretory indexes were calculated using the results of OGTT.

\section{Results}

Table 1 shows baseline characteristics of subjects in our study. Average age was $54.6 \pm 11.8$ years and $41.5 \%$ of the subjects were male.

Table 1. Baseline characteristics of the study subjects

\begin{tabular}{|c|c|c|c|c|c|}
\hline & $\begin{array}{c}\text { All } \\
(\mathrm{N}=388) \\
\end{array}$ & $\begin{array}{c}\text { NGT } \\
(\mathrm{N}=33)\end{array}$ & $\begin{array}{c}\text { Prediabetes } \\
(\mathrm{N}=153)\end{array}$ & $\begin{array}{c}D M \\
(N=202)\end{array}$ & $P$-value \\
\hline Age (yrs) & $54.6 \pm 11.8$ & $49.1 \pm 10.7$ & $52.9 \pm 10.9$ & $56.9 \pm 12.1$ & $<0.001$ \\
\hline Male [n (\%)] & 161 (41.5\%) & $6(18.2 \%)$ & 57 (37.3\%) & $98(48.5 \%)$ & 0.002 \\
\hline BMI $\left(\mathrm{kg} / \mathrm{m}^{2}\right)$ & $25.1 \pm 3.3$ & $23.8 \pm 3.2$ & $25.3 \pm 3.4$ & $25.1 \pm 3.2$ & 0.049 \\
\hline $\begin{array}{l}\text { Fasting glucose } \\
(\mathrm{mg} / \mathrm{dL})\end{array}$ & $125.8 \pm 40.0$ & $92.8 \pm 5.2$ & $106.7 \pm 7.8$ & $145.8 \pm 46.6$ & $<0.001$ \\
\hline $\begin{array}{l}\text { Fasting insulin } \\
\text { (ulU/mL) }\end{array}$ & $11.0 \pm 4.9$ & $8.99 \pm 2.98$ & $10.5 \pm 3.7$ & $11.7 \pm 5.8$ & 0.011 \\
\hline $\begin{array}{l}\text { Fasting C-peptide } \\
\text { (ng/mL) }\end{array}$ & $2.45 \pm 1.09$ & $1.55 \pm 0.61$ & $2.28 \pm 0.86$ & $2.73 \pm 1.20$ & $<0.001$ \\
\hline HbA1c (\%) & $6.64 \pm 1.65$ & $5.54 \pm 0.33$ & $5.78 \pm 0.44$ & $7.32 \pm 1.90$ & $<0.001$ \\
\hline $\begin{array}{l}\text { Intact proinsulin } \\
\text { (pmol/L) }\end{array}$ & $6.42 \pm 5.67$ & $2.96 \pm 1.57$ & $5.67 \pm 5.44$ & $7.56 \pm 5.95$ & $<0.001$ \\
\hline HOMA IR & $3.46 \pm 2.17$ & $2.07 \pm 0.73$ & $2.78 \pm 1.04$ & $4.21 \pm 2.63$ & $<0.001$ \\
\hline HOMA beta & $76.5 \pm 38.0$ & $111.1 \pm 37.8$ & $88.6 \pm 31.2$ & $61.7 \pm 35.9$ & $<0.001$ \\
\hline Matsuda index & $4.08 \pm 1.96$ & $6.08 \pm 2.35$ & $4.32 \pm 1.85$ & $3.57 \pm 1.72$ & $<0.001$ \\
\hline Stumvoll index & $6.72 \pm 2.37$ & $9.41 \pm 1.29$ & $7.46 \pm 2.15$ & $5.71 \pm 2.11$ & $<0.001$ \\
\hline OGIS & $349.8 \pm 64.7$ & $436.6 \pm 44.7$ & $380.2 \pm 43.4$ & $312.6 \pm 54.6$ & $<0.001$ \\
\hline IGI & $0.44 \pm 0.54$ & $0.91 \pm 0.76$ & $0.63 \pm 0.66$ & $0.21 \pm 0.18$ & $<0.001$ \\
\hline Disposition index & $1.67 \pm 2.13$ & $4.90 \pm 3.50$ & $2.32 \pm 2.10$ & $0.64 \pm 0.48$ & $<0.001$ \\
\hline
\end{tabular}

All data are presented as the means \pm SD.

P-value was calculated using Kruskall-Wallis test or $\chi 2$ test.

$\mathrm{BMI}$, body mass index; IGI, Insulinogenic index

Table 2. Spearman correlations of intact proinsulin with insulin resistance and sensitivity indexes $(A)$ and with insulin secretory indexes (B)

\begin{tabular}{|c|c|c|c|c|c|c|c|c|}
\hline \multirow[t]{2}{*}{ (A) } & \multicolumn{2}{|c|}{ HOMA-IR } & \multicolumn{2}{|c|}{ Matsuda index } & \multicolumn{2}{|c|}{ Stumvoll index } & \multicolumn{2}{|l|}{ OGIS } \\
\hline & $r$ & $\boldsymbol{P}$ & $r$ & $P$ & $r$ & $\boldsymbol{P}$ & $r$ & $\boldsymbol{P}$ \\
\hline $\begin{array}{l}\text { Intact } \\
\text { proinsulin }\end{array}$ & 0.504 & $<0.001$ & -0.445 & $<0.001$ & -0.449 & $<0.001$ & -0.399 & $<0.001$ \\
\hline \multirow[t]{2}{*}{$\overline{(B)}$} & \multicolumn{2}{|c|}{ HOMA-beta } & \multicolumn{2}{|l|}{ IGI } & \multicolumn{2}{|c|}{ Disposition index } & & \\
\hline & $r$ & $P$ & $r$ & $\boldsymbol{P}$ & $r$ & $P$ & & \\
\hline $\begin{array}{l}\text { Intact } \\
\text { proinsulin }\end{array}$ & 0.008 & 0.880 & -0.100 & 0.049 & -0.297 & $<0.001$ & & \\
\hline
\end{tabular}

Intact proinsulin level was positively correlated with homeostasis model assessment insulin resistance (HOMA-IR) $(r=0.504, P<0.001)$ and was inversely correlated with insulin sensitivity indexes (Matsuda index: $r=-$ 0.445, $P<0.001$; Stumvoll index: $r=-0.449, P<0.001$; OGIS: $r=-0.399$, $P<0.001$ ) (Table $2 A$, Figure 1 ). However, there were no significant correlations between intact proinsulin and insulin secretory indexes (HOMA-beta) (Table 2B).
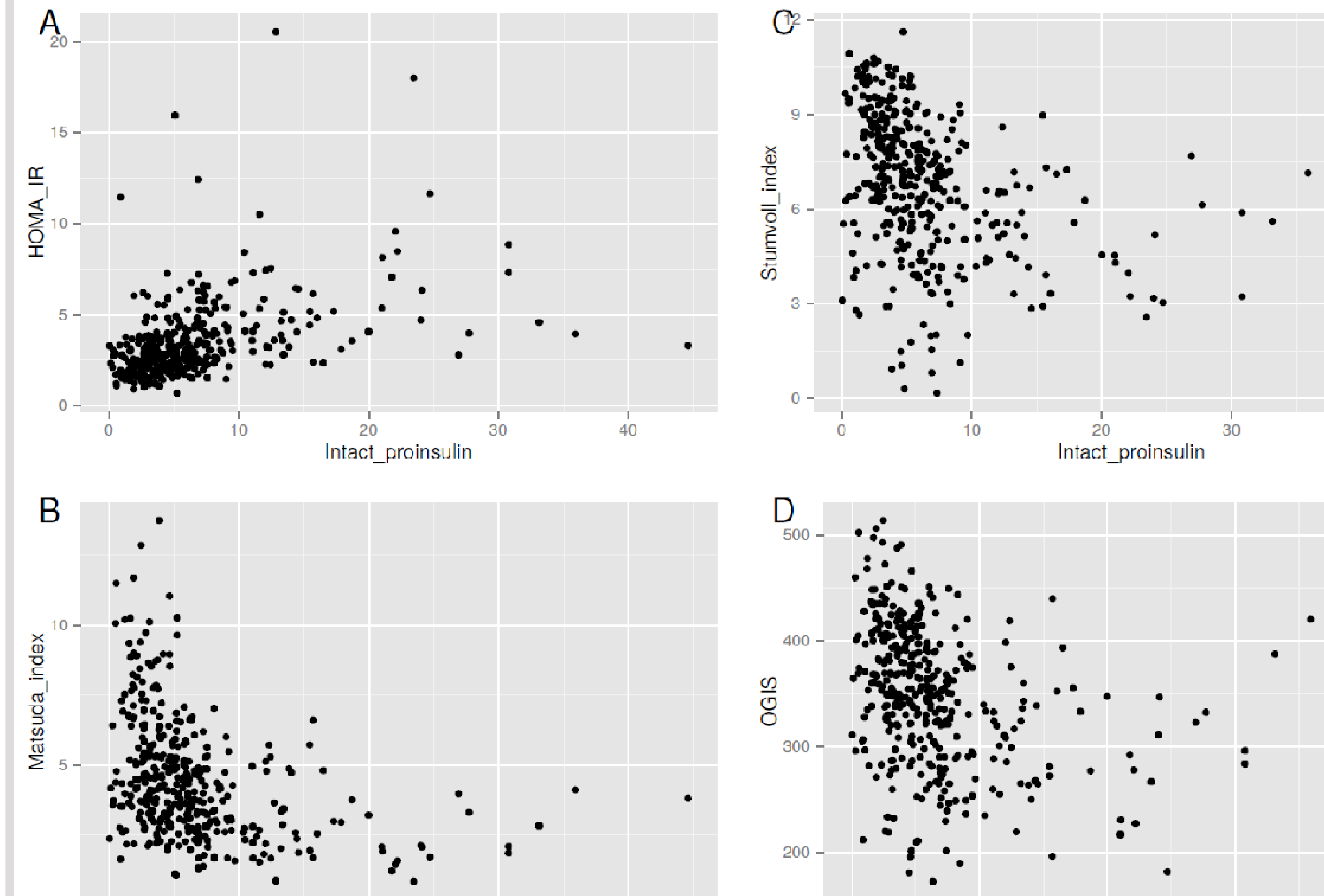

Figure 1. Correlation between intact proinsulin and insulin resistance or sensitivity indexes.

Total subjects were stratified by glucose tolerance status; normal glucose tolerance (NGT, $n=33$ ), prediabetes (impaired fasting glucose and/or impaired glucose tolerance, $n=153)$, and diabetes mellitus ( $D M, n=202$ ) (Table 1). Intact proinsulin level showed an increasing tendency with the deterioration of glucose tolerance $(P<0.001$, Table 1$)$. In the NGT group, intact proinsulin level was not correlated with both insulin sensitivity and secretory indexes (Table 3). However, in the prediabetes and DM groups, intact proinsulin levels were inversely correlated with insulin sensitivity indexes (Matsuda index, Stumvoll index, and OGIS index) and positively correlated with insulin secretory index (HOMA-beta) (Table 3).

Table 3. Spearman correlations of intact proinsulin with insulin resistance and sensitivity indexes $(A)$ and with insulin secretory indexes (B) in subgroups stratified by glucose tolerance status

\begin{tabular}{|c|c|c|c|c|c|c|c|c|}
\hline \multirow{2}{*}{$\begin{array}{l}\text { (A) } \\
\text { Intact } \\
\text { proinsulin }\end{array}$} & \multicolumn{2}{|c|}{ HOMA-IR } & \multicolumn{2}{|c|}{ Matsuda index } & \multicolumn{2}{|c|}{ Stumvoll index } & \multicolumn{2}{|l|}{ OGIS } \\
\hline & $r$ & $P$ & $r$ & $P$ & $r$ & $\boldsymbol{P}$ & $r$ & $P$ \\
\hline NGT & 0.193 & 0.282 & -0.261 & 0.143 & -0.212 & 0.237 & -0.469 & 0.006 \\
\hline Prediabetes & 0.405 & $<0.001$ & -0.389 & $<0.001$ & -0.400 & $<0.001$ & -0.231 & 0.004 \\
\hline DM & 0.472 & $<0.001$ & -0.389 & $<0.001$ & -0.301 & $<0.001$ & -0.255 & $<0.001$ \\
\hline (B) & HOMA & eta & IGI & & Dispos & on index & & \\
\hline $\begin{array}{l}\text { Intact } \\
\text { proinsulin }\end{array}$ & $r$ & $\boldsymbol{P}$ & $r$ & $\boldsymbol{P}$ & $r$ & $\boldsymbol{P}$ & & \\
\hline NGT & -0.146 & 0.419 & 0.122 & 0.498 & -0.077 & 0.669 & & \\
\hline Prediabetes & 0.248 & 0.002 & 0.082 & 0.314 & -0.134 & 0.098 & & \\
\hline DM & 0.163 & 0.020 & 0.113 & 0.112 & -0.105 & 0.139 & & \\
\hline
\end{tabular}

\section{Conclusions}

Intact proinsulin level increases with the deterioration of glucose tolerance and is significantly correlated with insulin resistance indexes but not with insulin secretory indexes in subjects with abnormal glucose tolerance. Moreover, intact proinsulin partly reflects beta cell function. 